\title{
Effect of Crystalline Relaxation on Fatigue Behavior of the Oriented High-Density Polyethylene Based on Nonlinear Viscoelastic Measurements
}

\author{
Nam-Ju Jo, Atsushi Takahara, and Tisato KajIYAma* \\ Department of Chemical Science \& Technology, Faculty of Engineering, \\ Kyushu University, 6-10-1, Hakozaki, Higashi-ku, Fukuoka 812, Japan
}

(Received January 5, 1994)

\begin{abstract}
Oriented high-density polyethylene (HDPE) specimens were prepared by drawing the HDPE film up to the draw ratio of 5.3 at $353 \mathrm{~K}$ corresponding to the crystalline relaxation temperature. Nonlinear viscoelasticity and higher-order structural change of the oriented HDPE during the fatigue process were investigated at various ambient temperatures. Nonlinear viscoelasticity of the oriented HDPE was estimated on the basis of nonlinear viscoelastic parameter (NVP). The magnitude of NVP at the $\alpha_{1}$-crystalline relaxation (intermosaic block crystalline relaxation) temperature region was lower than that at room temperature, since deformation in the intermosaic block region preferentially occurred in addition to that in the amorphous region. However, the fatigue strength at the $\alpha_{1}$-crystalline relaxation temperature was lower than that at the other ambient temperatures, because deformation in the intermosaic block region was very sensitive to strain or stress concentration. Since both lamellar crystalline and amorphous regions were deformed at the $\alpha_{2}$-crystalline relaxation temperature region, the magnitude of NVP became lower than that at the $\alpha_{1}$-crystalline relaxation temperature. Also, the fatigue strength at the $\alpha_{2}$-crystalline relaxation temperature was greater than that at the $\alpha_{1}$-crystalline relaxation temperature due to a more homogeneous deformation of the specimen.

KEY WORDS Oriented High-Density Polyethylene / Nonlinear Viscoelasticity / Fatigue / Nonlinear Viscoelastic Parameter / Higher-Order Structural Change / Mechanical Crystalline Relaxation /
\end{abstract}

Fatigue strength is a very important engineering property for safety assessment of structural materials. Under the condition which ultimately leads to fatigue failure, nonlinear viscoelastic behavior being accompanied with an irreversible structural change becomes prominent. Therefore, it may be reasonable to consider that the appearance of nonlinear viscoelasticity is closely related to fatigue failure.

The relationships between fatigue behavior and nonlinear viscoelasticity on the basis of the hysteresis energy loss for plasticized poly(vinyl chloride) (p-PVC), ${ }^{1}$ unoriented high-density polyethylene (HDPE), ${ }^{2}$ oriented HDPE, ${ }^{3,4}$ and short glass-fiber reinforced composite ${ }^{5}$ have been investigated by the authors. It has been revealed that the fatigue lifetimes of solid polymers decreased with an increase in the contribution of nonlinear viscoelasticity estimated from hysteresis energy loss. Also, the nonlinear viscoelastic parameter, NVP, was evaluated on the basis of Fourier analysis of higher-order stress wave. The physical importance of NVP under cyclic fatigue was discussed for the annealed and isothermally crystallized HDPEs, and it was concluded that the magnitudes of NVP for the annealed and the

* To whom correspondence should be addressed. 
isothermally crystallized HDPEs were closely related to both the magnitude of spherulite deformation and the degree of strain or stress concentration at the spherulite or crystallite interface. $^{6}$

The fatigue strength of polymeric materials was strongly influenced by the surrounding conditions, such as environmental media, temperature, humidity, and so on. Ambient temperature is one of the most important parameter, since thermal molecular motion of polymeric materials strongly depends on temperature. The $\alpha_{\mathrm{c}}$-relaxation behavior of PE is divided into the $\alpha_{1}$ - and $\alpha_{2}$-crystalline relaxation processes. In the $\alpha_{1}$-crystalline relaxation region, the intermosaic block region was preferentially deformed upon the application of external strain or stress, whereas in the $\alpha_{2}$ relaxation region, the uniform deformation of lamellar crystal occurred. ${ }^{7-9}$ Figure 1 shows the schematic representation of the $\beta-, \alpha_{1}-$, and $\alpha_{2}$-relaxation mechanisms. ${ }^{7-9}$ In the $\beta$-relaxation temperature range ( $c a .253 \mathrm{~K}$ ) being in relation to micro-Brownian motion of interlamellar amorphous chains, interlamellar slip and/or deformation of interlamellar amorphous region mainly occurs. Since deformation in the intermosaic block region is preferentially manifested in the $\alpha_{1}$-crystalline relaxation temperature region, the intermosaic region is sensitive to static or dynamic deformation.
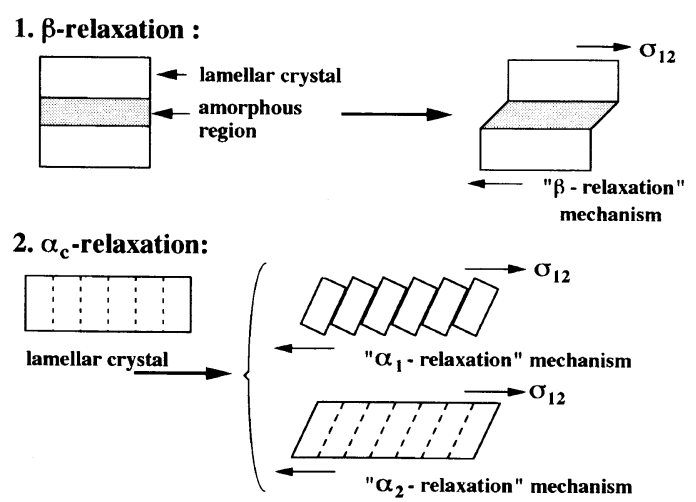

Figure 1. Schematic representation of $\beta-, \alpha_{1^{-}}$, and $\alpha_{2}$-relaxation mechanisms.
Then, microcrazes or microcracks may be preferentially initiated from the mosaic block boundary without any deformation in the mosaic block core. In the $\alpha_{2}$-crystalline relaxation temperature region, uniform shear deformation of lamellar crystals occurs in addition to their decomposition into large mosaic blocks. Fatigue strength of an ultradrawn sample of ultra-high molecular weight polyethylene (UHMWPE) was studied at various ambient temperatures. The fatigue strength of oriented UHMWPE at the $\alpha_{1}$ crystalline relaxation temperature was lower than those at room temperature and the $\alpha_{2}$ crystalline relaxation temperature, because imposed stress or strain is preferentially concentrated in the intermosaic block region at this temperature region. ${ }^{10}$

In this study, the effect of mechanical crystalline relaxation on fatigue behavior of an oriented HDPE drawn at the crystalline relaxation temperature has been investigated on the basis of nonlinear viscoelasticity and higher-order structural change measurements during the fatigue process. Also, the origin of nonlinear viscoelasticity under cyclic fatigue has been discussed on the basis of the analysis of higher-order structural change.

\section{EXPERIMENTAL}

\section{Specimens}

The polymer used in this study was a highdensity polyethylene (HDPE, Hizex 7000F, Mitsui Petrochemical Co., Ltd.) with $M_{w}=$ 260,000 . The drawing experiments were carried out at $353 \mathrm{~K}$ which corresponded to the crystalline relaxation temperature of HDPE. ${ }^{7-9}$ The draw ratio of 5.3 was defined as the ratio of the cross sectional areas of the sample before and after drawing.

\section{Fatigue Test and Estimation of Nonlinear Vis- coelasticity}

Figure 2 shows the blockdiagram of fatigue tester to investigate nonlinear dynamic visco- 


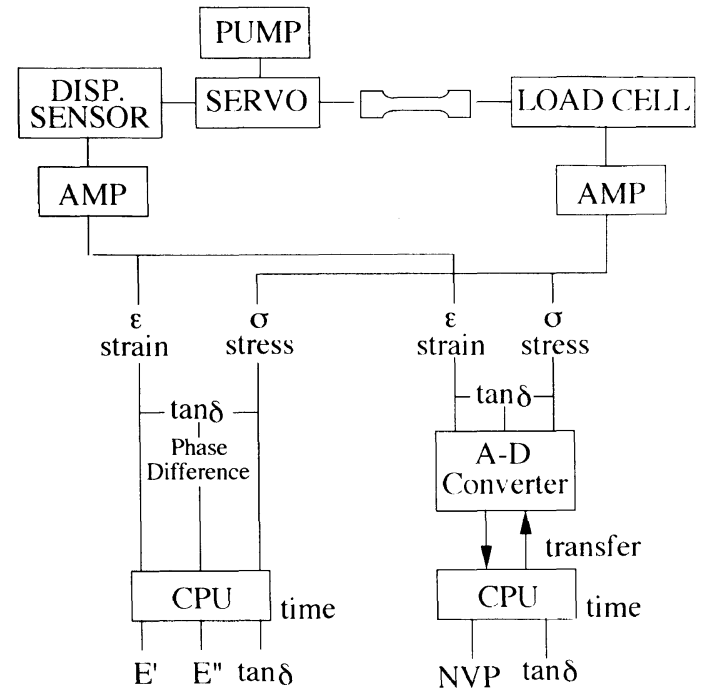

Figure 2. Blockdiagram of fatigue tester for investigation of nonlinear dynamic viscoelastic properties under cyclic fatigue.

elastic properties. The fatigue test was carried out using a fatigue tester which enabled continuous measurement of dynamic viscoelastic functions during the fatigue process. The strain and stress signals were measured by the semiconductor displacement sensor and load cell, respectively. The magnitude of apparent mechanical loss tangent, $\tan \delta$ was obtained from the phase difference between the stress and strain signals under cyclic straining. Also, the magnitude of apparent dynamic modulus was calculated from the amplitudes of the stress and strain signals. Fatigue tests were performed under the conditions of the testing frequency of $10 \mathrm{~Hz}$, and initial static tensile stress of $40 \mathrm{MPa}$. The imposed strain and response stress signals were collected with an AD converter. Since the A-D converter has a large memory, this can be used as a digital storage memory. The digital data were transferred to the personal computer, and the degree of nonlinear viscoelasticity was estimated on the basis of the nonlinear viscoelastic parameter, $\mathrm{NVP}^{6}$ being defined as follows:

$$
\begin{aligned}
& \mathrm{NVP}= \\
& \frac{\sqrt{a_{2}^{2}+b_{2}^{2}}+\sqrt{a_{3}^{2}+b_{3}^{2}}+\cdots \sqrt{a_{10}^{2}+b_{10}^{2}}}{\sqrt{a_{1}^{2}+b_{1}^{2}}}
\end{aligned}
$$

where $a_{n}$ and $b_{n}(n=1,2,3, \cdots)$ were the coefficients of Fourier series of stress signal.

\section{Wide Angle X-Ray Diffraction}

The change of crystalline state of samples during the fatigue process was investigated on the basis of wide angle $\mathrm{X}$-ray studies at room temperature using an $\mathrm{X}$-ray diffractometer with a rotating anode source $(40 \mathrm{kV}, 200 \mathrm{~mA})$ (RU-300: Rigaku Co., Ltd.). Assuming that the distribution function for lattice distortions and the shape factor are Lorentzian, the crystal size, $D_{h k l}$ and the lattice distortion, $g_{h k l}$ were evaluated on the basis of Hosemann's paracrystal analysis. ${ }^{11}$

\section{Small Angle X-Ray Scattering}

In order to evaluate the magnitude of long period and SAXS peak intensity for the unfatigued and fatigued samples, small angle $\mathrm{X}$-ray scattering profiles were obtained with $\mathrm{Ni}$-filtered $\mathrm{Cu}-K_{\alpha}$ radiation generated by $\mathrm{RU}$ 200 (Rigaku Co., Ltd.) using position sensitive proportional counter (PSPC) as a scattering photon counter. SAXS peak intensity gives the information about both the density difference between the crystalline and amorphous regions and the crystallinity. SAXS peak intensity was corrected by the thickness of sample.

\section{RESULTS AND DISCUSSION}

\section{Crystalline State of the Oriented HDPE Drawn at Crystalline Relaxation Temperature}

Table I shows the crystallite sizes, $D_{h k l}$, lattice distortion factors, $g_{h k l}$ in 110 and 200 directions for the unoriented and oriented HDPEs. Crystallite sizes and lattice distortion factors of the oriented HDPE were smaller and larger than those of the unoriented HDPE, respect- 
Table I. Crystal structural parameters of the unoriented and oriented HDPEs

\begin{tabular}{|c|c|c|c|c|}
\hline \multirow{2}{*}{ Sample } & $D_{110}$ & $D_{200}$ & $g_{110}$ & $g_{200}$ \\
\hline & $\mathrm{nm}$ & $\mathrm{nm}$ & $\%$ & $\%$ \\
\hline Unoriented & 20.5 & 18.6 & 0.85 & 1.83 \\
\hline Oriented at $535 \mathrm{~K}$ & 11.3 & 9.4 & 1.74 & 1.55 \\
\hline
\end{tabular}

ively. Peterlin reported the similar results, ${ }^{12,13}$ and Ishikawa et al. revealed that the long period of polyethylene single crystal became smaller by drawing due to the partial-compensating thickening effect. ${ }^{14}$ Also, Suehiro et al. studied extensively about the change of mosaic block size of polyethylene in a drawing process, and revealed that the mosaic block size strikingly decreased above the draw ratio of 5.0. ${ }^{15}$ Thus, it is reasonable to consider that the decrease in crystal dimensions, $D_{110}$ and $D_{200}$ by drawing may be strongly related to the formation of fibrillar structure which is generated from the tilting and slipping of lamellae before necking, resulting in the destruction of lamellae into folded chain blocks (mosaic blocks). ${ }^{7-9}$

Nonlinear Viscoelasticity and Higher-Order Structural Change of the Oriented HDPE during the Fatigue Process under Various Ambient Temperatures

Figure 3 shows the temperature dependences of the magnitude of dynamic storage modulus, $E^{\prime}$, mechanical loss tangent, $\tan \delta$, and nonlinear viscoelastic parameter, NVP, as well as the magnitude of higher-order ( $n$th order) harmonics of stress signal, $\left(\sqrt{a_{n}^{2}+b_{n}^{2}} / \sqrt{a_{1}^{2}+b_{1}^{2}}\right)$ for the drawn HDPE at $353 \mathrm{~K}$, of which data were obtained after cyclic fatigue for $100 \mathrm{~s}$ from the starting of fatigue test at $\varepsilon_{\mathrm{d}}=3.0 \%$. The magnitude of higher-order harmonics was obtained from the ratio of the root mean square of the $n$th harmonics coefficients to that of fundamental component coefficients. The magnitude of $E^{\prime}$ decreased slightly in a temperature range corresponding to the $\alpha_{1}$-crystalline

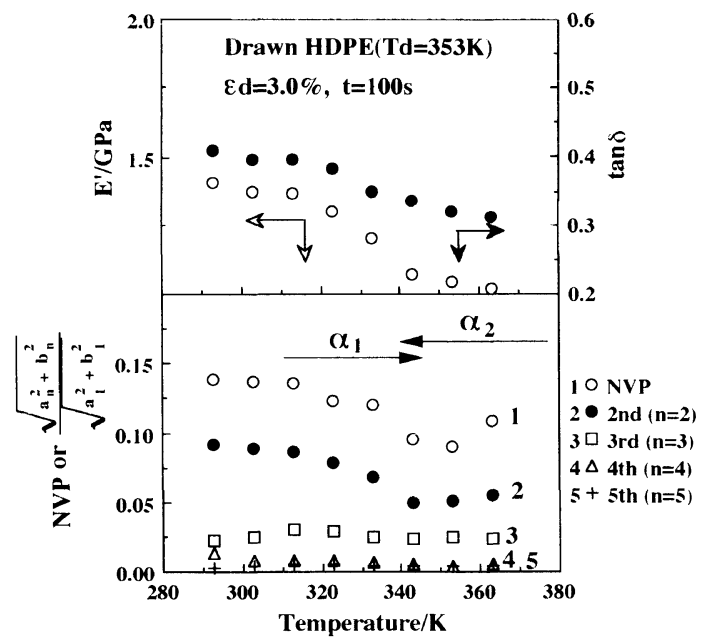

Figure 3. Temperature dependences of the magnitude of $E^{\prime}, \tan \delta$, and NVP, as well as the magnitude of higher-order ( $n$th order) harmonics of stress signal, $\left(\sqrt{a_{n}^{2}+b_{n}^{2}} / \sqrt{a_{1}^{2}+b_{1}^{2}}\right)$ for the drawn HDPE at $353 \mathrm{~K}$ in the case of cyclic fatigue after $100 \mathrm{~s}$ from the start of fatigue test at $\varepsilon_{\mathrm{d}}=3.0 \%$.

relaxation temperature, and then, decreased abruptly above around $330 \mathrm{~K}$. This might correspond to the increase of viscous component in the crystalline region with temperature, due to a considerable increase in thermal molecular motion in the crystalline region. In spite of the increase of viscous component in the crystalline region, $\tan \delta$ and NVP decreased slightly with temperature. Therefore, it might be reasonable to consider that the decrease of magnitude of $\tan \delta$ with temperature is attributed to the decrease in the contribution of nonlinear viscoelasticity. Also, the magnitudes of NVP and 2nd order harmonics decreased around $320 \mathrm{~K}$ corresponding to the $\alpha_{1}$-crystalline relaxation temperature. In a $\alpha_{1}$-crystalline relaxation temperature region, the deformation in the intermosaic block region compensates an imposed strain in addition to that in the interlamellar amorphous region. This indicates that the degree of deformation concentration in the amorphous region decreased in the $\alpha_{1}$-crystalline relaxation temperature, resulting in an apparently more homogeneous deformation. Then, the magni- 
tudes of NVP and 2nd order harmonics were lowered at around the $\alpha_{1}$-crystalline relaxation temperature region. In a temperature range of the $\alpha_{2}$-crystalline relaxation above around $340 \mathrm{~K}$, the magnitudes of NVP and 2nd order harmonics showed a remarkable decrease. This may arise from a more homogeneous deformation to cyclic straining because both amorphous and crystal regions can be simultaneously deformed under cyclic straining in this temperature region.

Figure 4 shows the plots of crystallite sizes, SAXS peak intensity, and long period for the drawn HDPE at $353 \mathrm{~K}$ against the ambient temperature. All the data were obtained after cyclic fatigue of $100 \mathrm{~s}$ from the start of fatigue test. As reported previously, ${ }^{8,9}$ an imposed

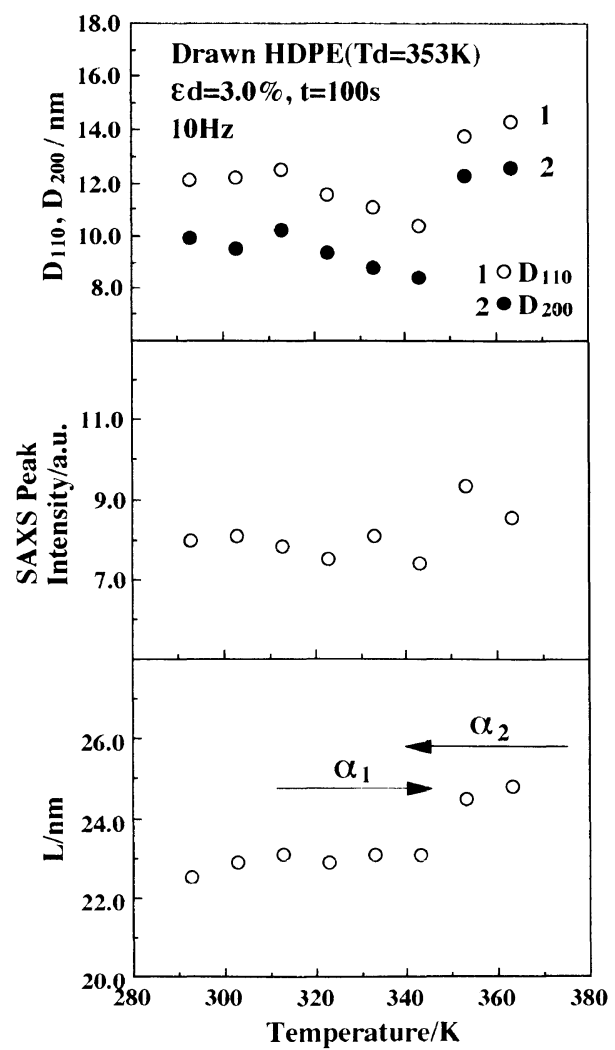

Figure 4. Plots of crystallite sizes, SAXS peak intensity, and long period for the drawn HDPE at $353 \mathrm{~K}$ in the case of cyclic fatigued after $100 \mathrm{~s}$ from the start of fatigue test at $\varepsilon_{\mathrm{d}}=3.0 \%$ against the ambient temperature. strain is concentrated in an interfacial mosaic block region in the case of a slight deformation or a cyclic fatigue tests, resulting in the decomposition into small fragments of mosaic blocks at around the $\alpha_{1}$-crystalline relaxation temperature. Also, Figure 4 exhibits a similar trend that mosaic block sizes, $D_{110}$ and $D_{200}$ became smaller after a cyclic fatigue test of $100 \mathrm{~s}$ at around the $\alpha_{1}$-crystalline relaxation temperature. However, the change in the SAXS peak intensity and long period were not remarkable. These results indicate that the decomposition of lamellar crystal into small mosaic block crystal preferentially occurred at the $\alpha_{1}$-crystalline relaxation temperature. On the other hand, in the case of a cyclic fatigue test above the $\alpha_{2}$-crystalline relaxation temperature, crystallite sizes, SAXS peak intensity, and long period became larger than the results fatigue-tested at the $\alpha_{1}$-crystalline relaxation temperature. This fact may arise from a more homogeneous sample deformation to cyclic straining, resulting in decomposition of lamellar crystals into large mosaic blocks in the case of a fatigue test above the $\alpha_{2}$-crystalline relaxation temperature.

\section{Imposed Strain Amplitude Dependence of Nonlinear Viscoelasticity and Structural Change for the Oriented HDPE at Various Ambient Temperatures}

Figure 5 shows the variations of $E^{\prime}, \tan \delta$, and NVP with imposed strain amplitudes, $\varepsilon_{\mathrm{d}}$ at ambient temperatures of $300 \mathrm{~K}$ and $353 \mathrm{~K}$ corresponding to the temperatures below and around the $\alpha_{2}$-crystalline relaxation temperatures, respectively. The magnitude of $E^{\prime}$ monotonously decreased, and that of $\tan \delta$ slightly increased with increasing the imposed strain amplitude. The magnitude of NVP as an index of nonlinear viscoelasticity abruptly increased with an increase in the magnitude of imposed strain amplitude. Therefore, the increase in $\tan \delta$ with the imposed strain amplitude might be due to the contribution of nonlinear viscoelasticity, because an increase 


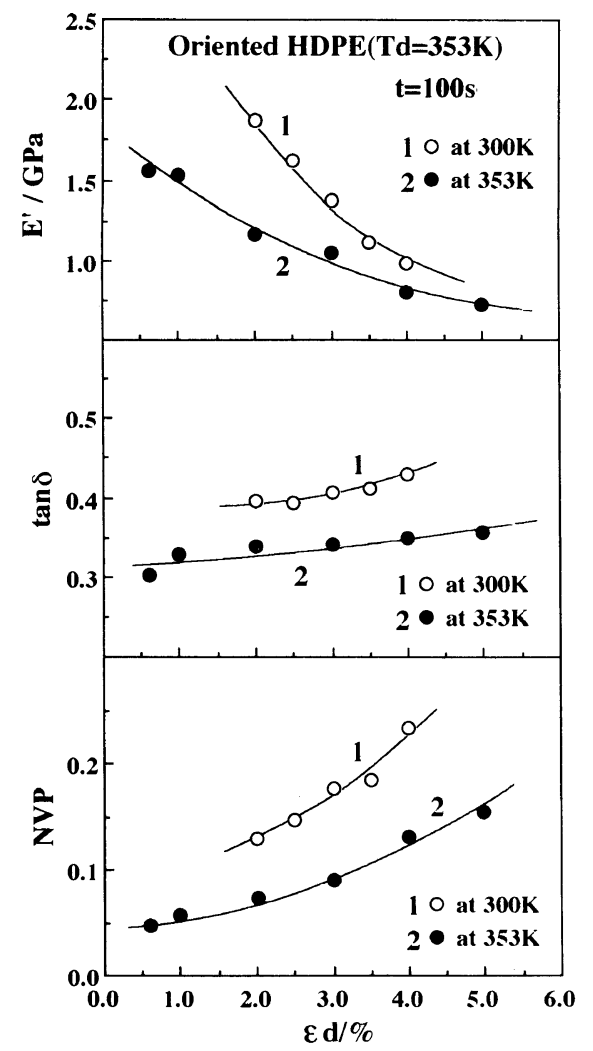

Figure 5. Variations of $E^{\prime}, \tan \delta$, and NVP with imposed strain amplitudes at the ambient temperatures of $300 \mathrm{~K}$ and $353 \mathrm{~K}$.

in viscous contribution to mechanical loss factor can be neglected due to almost constant specimen temperature during cyclic fatigue. An increase in nonlinear viscoelasticity will be discussed later on the basis of the higher-order structural change. The result that the magnitude of NVP at $353 \mathrm{~K}$ was smaller than that at $300 \mathrm{~K}$ can be explained in terms of the $\alpha_{2}$-mechanical crystalline relaxation mechanisms as shown in Figure 1. That is, since a more homogeneous sample deformation occurred in the case of a fatigue test at the $\alpha_{2}$ crystalline relaxation temperature, the limits of the imposed strain or stress range for the linear viscoelastic behavior of the drawn $\mathrm{HDPE}$ at $353 \mathrm{~K}$ increased.

Figure 6 summarizes the results of higher-

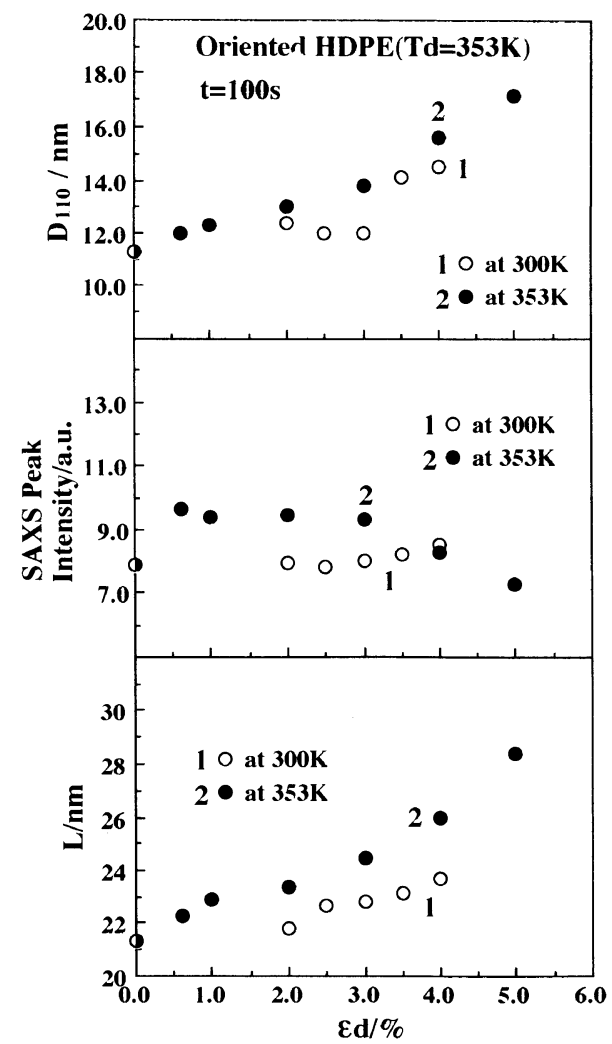

Figure 6. Plots of crystallite sizes, SAXS peak intensity, and long period for the drawn HDPE at $353 \mathrm{~K}$ with the magnitude of imposed strain after a cyclic fatigue test for $100 \mathrm{~s}$ at $300 \mathrm{~K}$ and $353 \mathrm{~K}$.

order structural changes, crystallite sizes, SAXS peak intensity, and long period, with the magnitude of imposed strain amplitude after a cyclic test for $100 \mathrm{~s}$ at $300 \mathrm{~K}$ and $353 \mathrm{~K}$. In the case of the ambient temperature of $300 \mathrm{~K}$, the magnitudes of crystallite size, SAXS peak intensity, and long period for the unfatigued and fatigued samples were comparable up to the imposed strain amplitude of $\varepsilon_{d}=3.0 \%$. That is, since the imposed cyclic straining is principally exerted in the amorphous region and/or crystallite boundary in the case of a cyclic fatigue test at $300 \mathrm{~K}$, it is reasonable to consider that the crystalline structural change induced by cyclic fatigue was not prominent. Also, the magnitude of NVP increased with the magnitude of imposed strain amplitude even if 
the applied strain amplitude was less than $3.0 \%$, as shown in Figure 5. Therefore, it is reasonably concluded that the nonlinear viscoelasticity originates apparently from the amorphous and/or crystallite boundary regions in the case of the oriented high-density polyethylene. On the other hand, the magnitudes of crystallite sizes and long period of the sample being fatigued at $353 \mathrm{~K}$ were larger than that at $300 \mathrm{~K}$. In the case of a cyclic fatigue test at $353 \mathrm{~K}$ corresponding to the $\alpha_{2}$-crystalline relaxation temperature, the limit of the imposed strain or stress range undergoing the linear viscoelastic behavior increase for the drawn HDPE at $353 \mathrm{~K}$ and the shear deformation of the crystalline lamellae occurs in addition to their decomposition into large crystalline blocks. ${ }^{7-9}$ Then, the magnitudes of crystallite sizes and long period in the case of a cyclic fatigue test at $353 \mathrm{~K}$ became larger than the test at $300 \mathrm{~K}$.

Also, in order to investigate whether the higher-order structural change originates from cyclic straining or initially applied static stress, the crystalline structural change under the application of constant stress was investigated. Figure 7 shows plots of crystallite size, $D_{110}$ of the specimens subjected to a certain tensile stress superimposed with dynamic strain (dynamic creep) and without dynamic strain

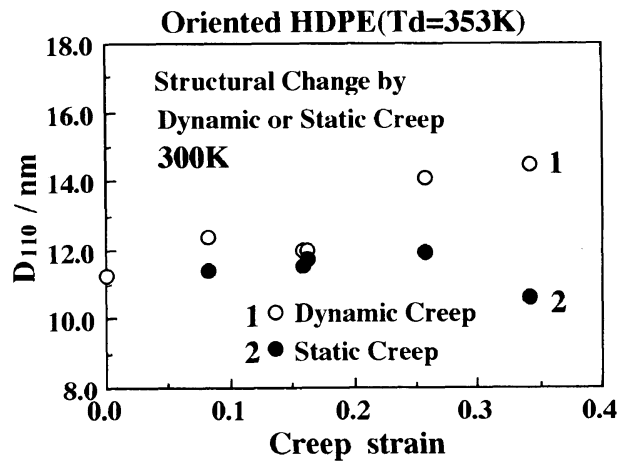

Figure 7. Plots of crystal structural parameters against creep strain for specimens subjected to certain tensile stress superimposed with dynamic strain (dynamic creep) and without dynamic strain (static creep). (static creep) at $300 \mathrm{~K} . D_{110}$ of specimen subjected to dynamic creep did not change in the case of the small magnitude of creep strain, and then, increased at the large magnitude of creep strain. On the other hand, $D_{110}$ of sample subjected to static creep did not change even at the large magnitude of creep strain. Therefore, it is apparent that the crystalline structural change under cyclic fatigue test arises from the dynamic cyclic straining.

\section{Variations of Nonlinear Viscoelasticity during} the Fatigue Process

Figure 8 shows the variations of $E^{\prime}, \tan \delta$, and NVP with time during the fatigue process for the oriented HDPE under the conditions

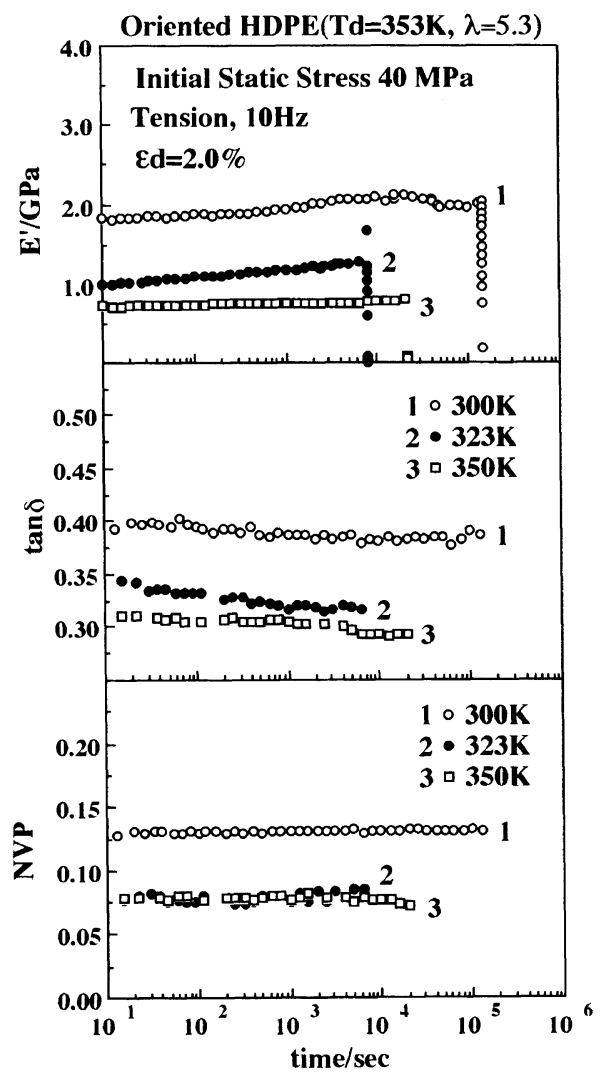

Figure 8. Variations of $E^{\prime}, \tan \delta$, and NVP for the drawn HDPE at $353 \mathrm{~K}$ with time during the fatigue process at $\varepsilon_{\mathrm{d}}=2.0 \%$ under the ambient temperatures of 300,323 , and $350 \mathrm{~K}$. 
of the imposed strain amplitude of $2.0 \%$ and the ambient temperatures of 300,323 , and $350 \mathrm{~K}$. The magnitudes of $E^{\prime}$ and $\tan \delta$ decreased with an increase in ambient temperature. The decrease in $\tan \delta$ with temperature is attributed to the decrease in nonlinear viscoelasticity. The magnitude of NVP in the case of a cyclic fatigue test at $323 \mathrm{~K}$ corresponding to the $\alpha_{1}$-crystalline relaxation temperature became smaller than that of the test at $300 \mathrm{~K}$. In a $\alpha_{1}$-crystalline relaxation temperature region, the intermosaic block region was deformed in addition to the amorphous region. This indicates that the degree of deformation concentration in the amorphous region decreased in this temperature region. Then, the magnitude of NVP at the $\alpha_{1}$-crystalline relaxation temperature became lower in comparison with that at $300 \mathrm{~K}$. The fatigue lifetime in the case of a cyclic fatigue test at $323 \mathrm{~K}$ became shorter than that at $300 \mathrm{~K}$, because the unstable intermosaic block region which can be the origin of crack growth was preferentially deformed by cyclic straining. Also, in order to investigate the relationship between the fatigue strength and the higher-order structural change under cyclic fatigue, crystalline states of fatigue fractured specimens were investigated. Table II summarizes fatigue lifetime and the crystal structural parameters for the drawn HDPEs after fatigue fracture in the cases of cyclic fatigue test at 300,323 , and $350 \mathrm{~K}$. Crystallite size, SAXS peak intensity and long period of fatigue fractured sample in the case of a cyclic fatigue test at $323 \mathrm{~K}$ corresponding to the $\alpha_{1}$-crystalline relaxation temperature became smaller than those at the other ambient tem- peratures. And, the lattice distortion factor in the case of a cyclic fatigue test at $323 \mathrm{~K}$ became larger than those at the other ambient temperatures. Therefore, it is reasonably concluded that the lowering of fatigue strength in the case of cyclic fatigue test at the $\alpha_{1}$ crystalline relaxation temperature results from the decomposition of lamellar crystals into small mosaic block crystals whose interfaces became the origin of crack growth. On the other hand, in the case of a cyclic fatigue test at $350 \mathrm{~K}$ corresponding to the $\alpha_{2}$-crystalline relaxation temperature region, since both the crystal and amorphous regions can be simultaneously deformed under cyclic fatigue, the magnitude of NVP became smaller than that at $300 \mathrm{~K}$. Also, the fatigue lifetime in the case of a cyclic fatigue test at $350 \mathrm{~K}$ became longer, and the crystallite size, SAXS peak intensity, and long period became larger than those of the test at the $\alpha_{1}$-crystalline relaxation temperature. Therefore, it is reasonably concluded that the increase in fatigue strength at the $\alpha_{2}$-crystalline relaxation temperature compared with the test at the $\alpha_{1}$-crystalline relaxation temperature results from a more homogeneous sample deformation.

In order to study the relationship between NVP and fatigue lifetime more in detail, the fatigue time dependences of higher harmonics of Fourier expanded stress signal were investigated. Figure 9 shows the time dependence of higher-order harmonics of stress signal for the drawn HDPE under the conditions of $\varepsilon_{\mathrm{d}}=2.0 \%$ and the ambient temperatures of 300 , 323 , and $350 \mathrm{~K}$. In spite of the different deformation mechanisms at each temperature,

Table II. Fatigue lifetime and crystal structural parameters of the oriented HDPEs after fatigue fracture at 300,323 , and $350 \mathrm{~K}$

\begin{tabular}{ccccccc}
\hline $\begin{array}{c}\text { Ambient } \\
\text { temperature }\end{array}$ & $\varepsilon_{\mathrm{d}} / \%$ & $t_{\mathrm{f}} / \mathrm{s}$ & $D_{110} / \mathrm{nm}$ & $g_{110} / \%$ & $\begin{array}{r}\text { SAXS Peak } \\
\text { intensity } / \text { a.u. }\end{array}$ & $L / \mathrm{nm}$ \\
\hline $300 \mathrm{~K}$ & 2.0 & 143500 & 12.5 & 1.49 & 7.93 & 22.7 \\
$323 \mathrm{~K}$ & 2.0 & 7900 & 10.9 & 1.52 & 7.61 & 20.9 \\
$350 \mathrm{~K}$ & 2.0 & 21600 & 13.4 & 1.34 & 8.82 & 23.4 \\
\hline
\end{tabular}


Effect of Crystalline Relaxation on Fatigue Behavior of the Oriented High-Density Polyethylene

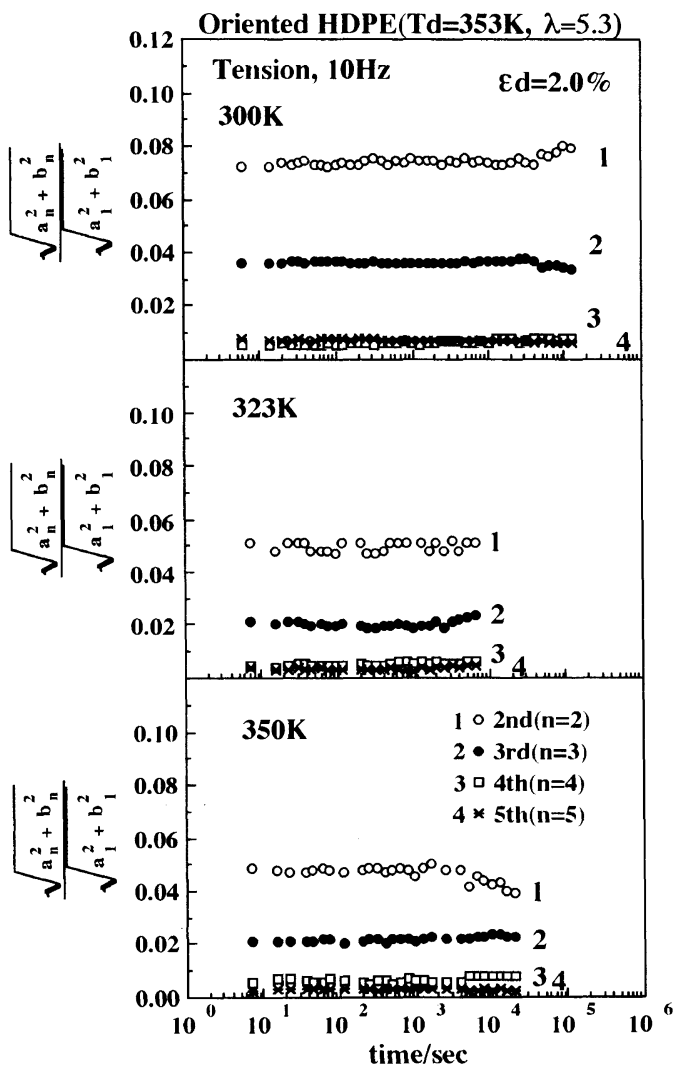

Figure 9. Time dependences of higher-order harmonics of stress signal for the drawn HDPE at $353 \mathrm{~K}$ during the fatigue process at the ambient temperatures of 300,323 , and $350 \mathrm{~K}$.

the magnitude of 2nd-order harmonics of Fourier series was greater than the other higher-order harmonics, and then, the 2ndorder harmonics was the greatest contribution to NVP. The next largest one was the 3rdorder harmonics. The magnitude of the other harmonics can be almost negligible in comparison with those of the 2nd and 3rd-order harmonics. Also, the magnitudes of higherorder harmonics in the cases of cyclic fatigue tests at $323 \mathrm{~K}$ and $350 \mathrm{~K}$ were smaller than the test at $300 \mathrm{~K}$. This may be explained by both a relatively larger homogeneous deformation in the crystalline region at $323 \mathrm{~K}$ and $350 \mathrm{~K}$ and also, a remarkable heterogeneous deformation in the interlamellar amorphous region at $300 \mathrm{~K}$.

\section{CONCLUSIONS}

Nonlinear viscoelasticity and higher-order structural change during the fatigue process under various ambient temperatures for the oriented HDPE drawn at the $\alpha_{2}$-crystalline relaxation temperature were investigated on the basis of NVP and X-ray study, respectively. It was revealed that nonlinear viscoelasticity during the fatigue process became prominent as the imposed strain amplitude increased. In the case of a cyclic fatigue test in the $\alpha_{1}$ crystalline relaxation temperature, since the degree of cyclic strain concentration in the amorphous region decreased because of the deformation in the intermosaic block region, the magnitude of NVP became smaller than the test at room temperature. Also, the fatigue lifetime was shorter than the tests at the other ambient temperatures, because lamellar crystals were decomposed into small mosaic block crystals whose interfaces became the origin of crack growth under cyclic straining. In the case of a cyclic fatigue test in the $\alpha_{2}$-crystalline relaxation temperature region, because of a more homogeneous sample deformation and the decrease in the degree of deformation concentration in the amorphous and/or crystallite boundary regions, the magnitude of NVP became smaller than the test at room temperature. The fatigue strength at the $\alpha_{2}$ crystalline relaxation temperature was greater than that at the $\alpha_{1}$-crystalline relaxation temperature due to the more homogeneous sample deformation.

\section{REFERENCES}

1. A. Takahara, K. Yamada, T. Kajiyama, and M. Takayanagi, J. Appl. Polym. Sci., 25, 597 (1980).

2. A. Takahara, K. Yamada, T. Kajiyama, and M. Takayanagi, J. Appl. Polym. Sci., 26, 1085 (1981).

3. N. Kaiya, M. Kugishima, A. Takahara, and T. Kajiyama, Sen-i Gakkaishi, 42, T-127 (1986).

4. N. Kaiya, A. Takahara, and T. Kajiyama, Polym. J., 
21, 523 (1989).

5. A. Takahara, T. Magome, and T. Kajiyama, $J$. Polym. Sci. Polym. Phys. Ed., 32, 839 (1994).

6. N.-J. Jo, A. Takahara, and T. Kajiyama, Polym. J., 25, 721 (1993).

7. T. Kajiyama, T. Okada, A. Sakoda, and M. Takayanagi, J. Macromol. Sci.-Phys., B7, 583 (1973).

8. T. Kajiyama, T. Okada, and M. Takayanagi, $J$. Macromol. Sci.-Phys., B9, 35 (1974).

9. T. Kajiyama and M. Takayanagi, J. Macromol. Sci.-Phys., B10, 131 (1974).

10. N. Kaiya, A. Takahara, and T. Kajiyama, Polym. J.,
22, 859 (1990).

11. R. Hosemann and W. Wilke, Makromol. Chem., 118, 230 (1968).

12. G. Meinel, N. Morosoff, and A. Peterlin, J. Polym. Sci., A-2, 8, 1723 (1970).

13. G. Meinel and A. Peterlin, J. Polym. Sci., A-2, 9, 67 (1971).

14. K. Ishikawa, K. Miyasaka, and M. Maeda, J. Polym. Sci., A-2, 7, 2029 (1969).

15. K. Suehiro, T. Terashima, and M. Takayanagi, $J$. Mater. Sci., 9, 1563 (1974). 